\title{
Oxylipid profiles of dairy cattle vary throughout the transition into early mammary gland involution
}

\author{
A. K. Putman, J. L. Brown, J. C. Gandy, A. Abuelo, and L. M. Sordillo ${ }^{1}$ \\ Department of Large Animal Clinical Sciences, College of Veterinary Medicine, Michigan State University, East Lansing 48824
}

\section{ABSTRACT}

Successful lactation in multiparous dairy cattle relies on a well-managed dry period that allows the mammary gland to remodel and regenerate between lactations. Oxylipids are potent inflammatory mediators that are capable of regulating all aspects of inflammation. Although an oxylipid profile has been documented for periparturient and lactating cattle, little work has been done to define the profile of cows in the early dry period. Therefore, our group aimed to characterize the oxylipid profile in healthy cows during the transition into early mammary gland involution. Plasma samples were collected from 10 healthy Holstein dairy cows via coccygeal venipuncture $6 \mathrm{~d}$ before dry-off $(\mathrm{d}-6)$, at dry-off $(\mathrm{d} 0)$, and $1(\mathrm{~d}+1), 2(\mathrm{~d}+2), 6(\mathrm{~d}+6)$, and $12(\mathrm{~d}$ +12 ) d after the dry-off date. Liquid chromatographymass spectrometry was used to quantify select monounsaturated fatty acids, polyunsaturated fatty acids, and saturated fatty acids, whereas oxylipids were quantified using liquid chromatography-tandem mass spectrometry. The results of this study revealed a unique profile of pro- and anti-inflammatory oxylipids throughout the transition from late lactation into the dry period. Many compounds reached the highest concentrations of the study at $\mathrm{d}+1, \mathrm{~d}+2$, or $\mathrm{d}+12$, whereas others reached the lowest concentrations at $d+12$. The characterization of this profile allows for further understanding of the physiology of early mammary involution. Future studies should investigate how the oxylipid profile of early mammary involution may affect the health and productivity of dairy cows.

Key words: dry cow, involution, fatty acid, oxylipin

\section{INTRODUCTION}

Within a typical lactation cycle, the period of time in which a cow is not producing milk is known as the dry period. During this critical stage, the mammary

Received June 4, 2018

Accepted November 15, 2018.

${ }^{1}$ Corresponding author: sordillo@msu.edu gland remodels and regenerates in preparation for the ensuing lactation. In fact, omitting the dry period can lead to production losses ranging from 12 to $25 \%$ in the subsequent lactation (Rastani et al., 2005). The first $15 \mathrm{~d}$ after dry-off, which are a part of acute involution, are particularly important to the health and productivity of the cow (Smith et al., 2017). During this time, the mammary gland is highly susceptible to new infections, partially because abrupt cessation of milking results in a relatively compromised streak canal that is no longer being flushed to aid in the removal of bacteria (Bradley and Green, 2004). Furthermore, early involution is coupled with major hormonal, nutritional, and physiological changes as the mammary gland undergoes a change from a lactating to nonlactating state. For example, the mammary gland becomes engorged with milk, leading to cattle discomfort and is infiltrated with immune cells to remove residual milk and debris during this time (Sordillo and Nickerson, 1988; Zobel et al., 2015). Recruitment of inflammatory cells to the mammary gland is one of several important processes involved in mammary tissue remodeling (Atabai et al., 2007) and depends upon an appropriate inflammatory response.

An important component of the innate immune response is inflammation. When regulated properly, inflammation is capable of resolving tissue insults, both infectious and otherwise, without causing further tissue damage. For instance, when mastitis-causing pathogens are recognized by the host, an immune response will occur that facilitates the neutralization of the organisms without compromising milk or tissues in a noticeable way (Sordillo, 2018). If inflammation is not controlled appropriately, however, mammary gland tissue damage can occur that may lead to negative effects on production. For example, the immune cells recruited during an inflammatory response can cause damage to mammary gland epithelial cells, leading to decreased milk production (Akers and Nickerson, 2011). Due to the potential damage inflammation can cause to host tissues, the onset and resolution of inflammation is tightly regulated and orchestrated by many mediators. One such class of mediators are oxylipids, which are 


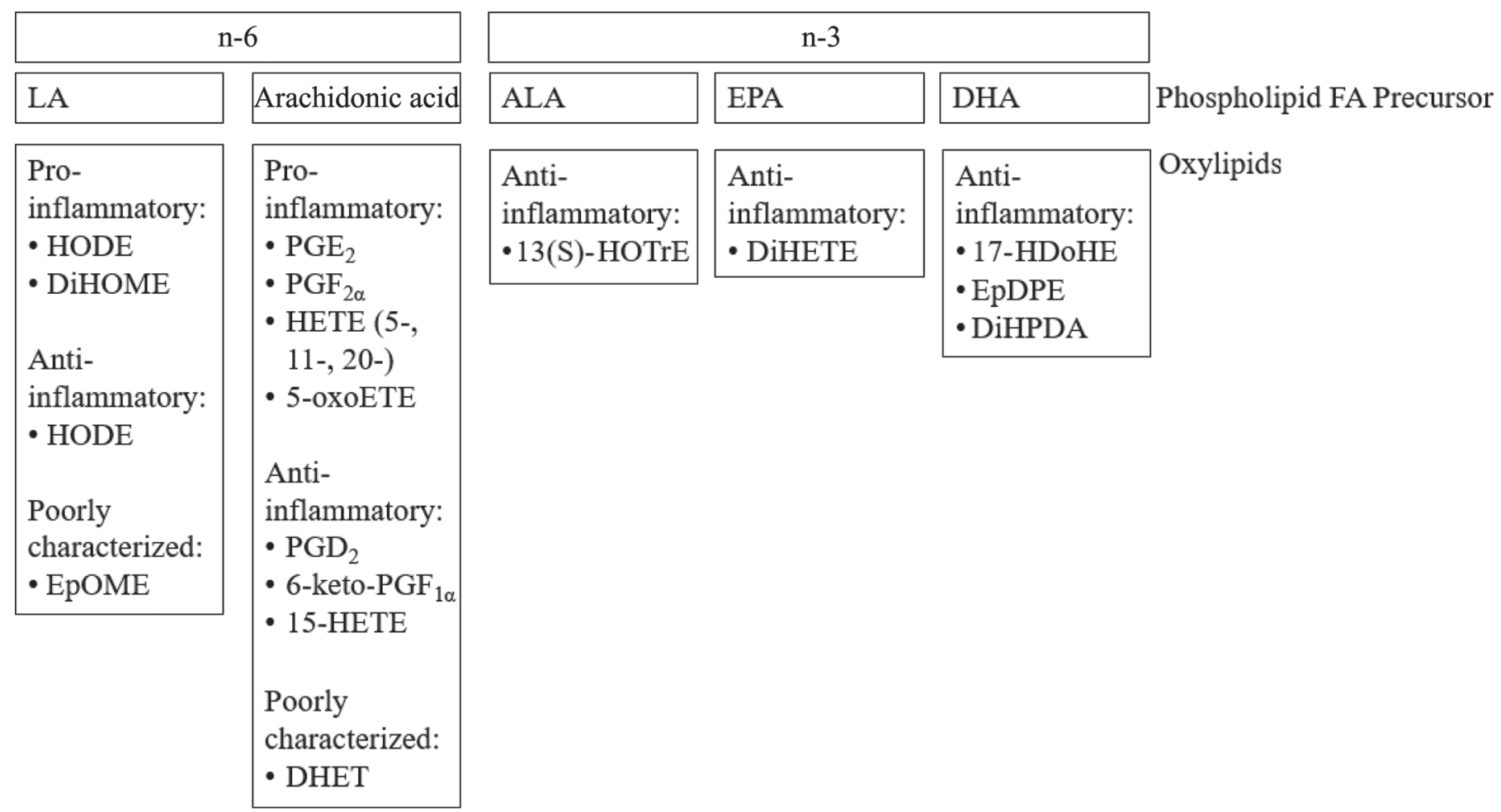

Figure 1. Sources and putative roles for oxylipids described in the present study. FA = fatty acid; LA = linoleic acid; ALA $=\alpha$-linolenic acid; EPA = eicosapentaenoic acid; DHA = docosahexaenoic acid; HODE = hydroxyoctadecadienoic acid; DiHOME = dihydroxyoctadecenoic acid; EpOME = epoxyoctadecaenoic acid; HETE = hydroxyeicosatetraenoic acid; oxoETE = oxoeicosatetraenoic acid; DHET $=$ dihydroxyeicosatrienoic acid; HOTrE = hydroxyoctadecatrienoic acid; DiHETE = dihydroxyeicosatetraenoic acid; HDoHE = hydroxydocosahexaenoic acid; $\mathrm{EpDPE}=$ epoxydocosapentaenoic acid; DiHPDA = dihydroperoxydocosahexaenoic acid.

derived from phospholipid membrane fatty acids (FA) via enzymatic or nonenzymatic pathways (Buczynski et al., 2009). Enzymatic pathways include cyclooxygenase (COX), lipoxygenase (LOX), and cytochrome-P450 (CYP), whereas nonenzymatic oxidation can occur when FA interact with reactive metabolites, such as reactive oxygen species (ROS; Buczynski et al., 2009). Oxylipids are capable of regulating all aspects of inflammation, possessing both pro-inflammatory and anti-inflammatory functions (Gabbs et al., 2015).

The FA substrate that oxylipids are derived from is a major determinant of their function. For instance, those that are derived from n-6 PUFA, such as arachidonic acid and linoleic acid ( $\mathbf{L A})$, tend to contribute to a pro-inflammatory state. On the other hand, those derived from n-3 PUFA, such as eicosapentaenoic acid (EPA) and docosahexaenoic acid (DHA), are generally considered anti-inflammatory (Calder, 2009). Products of PUFA peroxidation, including hydroperoxyoctadecadienoic acid and hydroperoxyeicosatetraenoic acid, typically are reduced immediately into more stable hydroxyls, such as hydroxyoctadecadienoic acid (HODE) and hydroxyeicosatetraenoic acid (HETE;
Raphael et al., 2014). Although some oxylipids act primarily as anti-inflammatory substances, such as those derived from n-3 PUFA, others may have multiple effects depending on cell type and location. For instance, HODE can be pro-inflammatory via adhesion molecule activation on endothelial cells (Friedrichs et al., 1999). However, HODE can also be anti-inflammatory by decreasing tumor necrosis factor- $\alpha$-induced inflammatory responses in human epithelial cells (Altmann et al., 2007). Sources of oxylipids and their putative roles are depicted in Figure 1.

The relative amount and potency of pro-inflammatory to anti-inflammatory oxylipids generates a profile that can influence inflammatory outcomes (Raphael et al., 2014). Previous work characterizing the complex oxylipid profile of dairy cattle has involved the periparturient and lactating cow during health and disease, such as mastitis. Given that cows entering the dry period experience a major physiological change like that of periparturient cows, it can be anticipated that the oxylipid profile of dry cows would also be complex in nature. However, the oxylipid profile of dairy cattle entering the early dry period has not been explored. De- 
termining the oxylipid profile of cows during this time provides further insight into the physiology of the dry period, leading to new hypotheses on how cow health and productivity may be affected. Therefore, this study aimed to document for the first time changes in these potent inflammatory mediators during the transition from late lactation to the early dry period.

\section{MATERIALS AND METHODS}

\section{Animals}

This study was approved by the Michigan State University Institutional Animal Care and Use Committee. Holstein cows $(n=10)$ from a commercial dairy herd were enrolled $56 \mathrm{~d}$ before expected calving date with owner consent, were free from clinical disease, and had SCC $<250 \times 10^{3}$ cells $/ \mathrm{mL}$ at their last DHIA test date. All cows were housed in a freestall barn, were grouped according to stage of lactation, and were milked 2 times/d before abrupt cessation of milking. At the time of dry-off, cows were treated with a standard intramammary antimicrobial treatment. Average milk production at the last DHIA test day was $31.7 \mathrm{~kg} / \mathrm{d}$ (range: $17.8-39.5 \mathrm{~kg} / \mathrm{d}$ ), and the average DIM was 315 (range: 291-357). The average BCS was 3.2 out of 5 (range: 2.7-3.9), and parity ranged from 1 to 5 (average $=2.8$ \pm 1.5 ). Cows had ad libitum access to a TMR and water. Animals sampled at $d-6$ were fed a late lactation diet, whereas those sampled for the remainder of the study were fed a far-off dry cow diet (Table 1). Cows were sampled between the summer and fall of 2017 .

\section{Sample Collection and Processing}

Blood samples were collected aseptically into EDTAcontaining evacuated tubes via coccygeal venipuncture between 0800 and $1000 \mathrm{~h}$. Sampling occurred at 6 time points: $\mathrm{d}-6,0,+1,+2,+6$, and +12 relative to the dry-off date. Samples were immediately stored on ice during transportation and processing.

Upon returning to the laboratory, blood samples were centrifuged at $1,449 \times g$ for $15 \mathrm{~min}$ at $4^{\circ} \mathrm{C}$, and plasma was subsequently harvested, aliquoted, and flash-frozen in liquid nitrogen. Plasma samples were stored at $-80^{\circ} \mathrm{C}$ pending analysis via liquid chromatography (LC)-MS or LC-MS/MS within 1 mo.

\section{Targeted Lipidomics}

Targeted MUFA, PUFA, and SFA were analyzed with LC-MS, and oxylipids were quantified using LC-MS/ MS (Table 2). Plasma samples $(1 \mathrm{~mL})$ were mixed with an antioxidant reducing agent mixture of $50 \%$ methanol, $25 \%$ ethanol, $25 \%$ water, butylhydroxytoluene (0.9 mM; Acros, Thermo Fisher Scientific, Waltham, MA), EDTA (0.54 mM; Sigma-Aldrich, St. Louis, MO), triphenylphosphine (3.2 $\mathrm{mM}$; Sigma-Aldrich), and indomethacin (5.6 mM; Cayman Chemical, Ann Arbor, MI) to prevent ex vivo lipid peroxidation and oxidation of preformed oxylipids (Mavangira et al., 2015).

Deuterated internal standards in the following concentrations were added to each sample: 5(S)hydroxyeicosatetraenoic acid- $d_{8}(0.25 \mu M), 15(\mathrm{~S})$-hydroxyeicosatetraenoic acid- $d_{8}(0.25 \mu M), 8(9)$-epoxye-

Table 1. Diet of late lactation and early dry cows for the study period on a DM basis

\begin{tabular}{|c|c|c|}
\hline Item & Late lactation & Far-off dry \\
\hline Ingredient & $\begin{array}{l}\text { Canola } \\
\text { Corn gluten } \\
\text { HMSC }^{1} \\
\text { Alfalfa } \\
\text { Corn silage } \\
\text { Mineral supplement }\end{array}$ & $\begin{array}{l}\text { Straw } \\
\text { Grass silage } \\
\text { Corn silage } \\
\text { Salt } \\
\text { Mineral supplement }\end{array}$ \\
\hline $\mathrm{NE}_{\mathrm{L}}^{2}(\mathrm{Mcal} / \mathrm{kg})$ & 1.5 & 1.15 \\
\hline $\mathrm{CP}(\%)$ & 19 & 11 \\
\hline Fat (\%) & 5.3 & 2.3 \\
\hline $\operatorname{NFC}(\%)$ & 38 & 17 \\
\hline Calcium (\%) & 0.75 & 0.51 \\
\hline Phosphorus (\%) & 0.69 & 0.36 \\
\hline Magnesium (\%) & 0.78 & 0.31 \\
\hline Potassium (\%) & 1.4 & 2.5 \\
\hline Sodium $(\%)$ & 0.29 & 0.34 \\
\hline Chloride (\%) & 0.53 & 0.99 \\
\hline Sulfur (\%) & 0.3 & 0.18 \\
\hline Selenium (mg/kg) & 6.2 & 23 \\
\hline Vitamin E (IU/animal) & 290 & 1,064 \\
\hline
\end{tabular}

${ }^{1} \mathrm{HMSC}=$ high-moisture shelled corn.

${ }^{2} \mathrm{NE}_{\mathrm{L}}=$ the estimated energy of lactation for an animal consuming $3 \times$ its requirement. 
icosatrienoic acid- $d_{11}(0.5 \mu M)$, prostaglandin $\mathrm{E}_{2}-d_{9}(0.5$ $\mu M)$, 8,9-dihydroxyeicosatrienoic acid- $d_{11}(0.25 \mu M)$, arachidonic acid- $d_{8}(50 \mu M), 2$-arachidonoyl glycerol- $d_{8}$ $(2 \mu M)$, and arachidonoyl ethanolamide- $d_{8}(0.25 \mu M)$ in the amount of $15 \mu \mathrm{L}$. For FA, solid phase extraction was carried out using Waters (Milford, MA) OASIS Prime HLB 3cc 150 mg Extraction Cartridges. Samples were passed through the columns, then washed with $5 \%$ methanol and eluted with acetonitrile:methanol (95:5). Solid phase extraction for oxylipids was carried out using Phenomenox (Torrance, CA) Strata-X 33 $\mu \mathrm{m}$ polymeric reversed-phase $200 \mathrm{mg} / 3 \mathrm{~mL}$ columns preconditioned with $3 \mathrm{~mL}$ of methanol followed by 3 $\mathrm{mL}$ of HPLC water. Supernatants were loaded into the columns, then washed with $20 \%$ methanol and eluted with a 50:50 mixture of methanol and acetonitrile with $2 \%$ formic acid. Removal of volatile solvents was performed with a Savant SpeedVac (Thermo Fisher

Table 2. Lipids and corresponding abbreviations analyzed in the study

\begin{tabular}{|c|c|}
\hline Lipid & Abbreviation \\
\hline Arachidonic acid & - \\
\hline Adrenic acid & - \\
\hline$\alpha$-Linolenic acid & ALA \\
\hline Docosahexaenoic acid & DHA \\
\hline Docosapentaenoic acid & DPA \\
\hline Eicosapentaenoic acid & EPA \\
\hline Dihomo- $\gamma$-linolenic acid & Dihomo-ALA \\
\hline Linoleic acid & LA \\
\hline Lauric acid & - \\
\hline Myristic acid & - \\
\hline Oleic acid & - \\
\hline Palmitic acid & - \\
\hline Palmitoleic acid & - \\
\hline Stearic acid & - \\
\hline Thromboxane $\mathrm{B}_{2}$ & $\mathrm{TXB}_{2}$ \\
\hline Prostaglandin $\mathrm{D}_{2}$ & - \\
\hline Prostaglandin $\mathrm{E}_{2}$ & - \\
\hline $\mathrm{PGF}_{2 \alpha}$ & $\mathrm{PGF}_{2 \alpha}$ \\
\hline 6-Keto-prostaglandin $\mathrm{F}_{1 \alpha}$ & - \\
\hline 5-Hydroxyeicosatetraenoic acid & 5-HETE \\
\hline 5-Oxoeicosatetraenoic acid & 5-oxoETE \\
\hline 8,9-Dihydroxyeicosatrienoic acid & 8,9-DHET \\
\hline 9,10 -Epoxyoctadecenoic acid & $9,10-\mathrm{EpOME}$ \\
\hline 9,10 -Dihydroxyoctadecenoic acid & 9,10-DiHOME \\
\hline 9-Hydroxyoctadecadienoic acid & 9 -HODE \\
\hline 9-Oxooctadecadienoic acid & 9-oxoODE \\
\hline 11,12-Dihydroxyeicosatrienoic acid & 11,12-DHET \\
\hline 11-Hydroxyeicosatetraenoic acid & 11-HETE \\
\hline 12,13-Epoxyoctadecenoic acid & 12,13-EpOME \\
\hline 12,13 -Dihydroxyoctadecenoic acid & 12,13 -DiHOME \\
\hline 13-Oxooctadecadienoic acid & 13-oxoODE \\
\hline 14,15-Dihydroxyeicosatetraenoic acid & 14,15-DiHETE \\
\hline 15-Hydroxyeicosatetraenoic acid & 15-HETE \\
\hline 17,18-Dihydroxyeicosatetraenoic acid & 17,18-DiHETE \\
\hline 20-Hydroxyeicosatetraenoic acid & 20-HETE \\
\hline 17-Hydroxydocosahexaenoic acid & 17-HDoHE \\
\hline 19,20-Dihydroperoxydocosahexaenoic acid & 19,20-DiHPDA \\
\hline 19,20-Epoxydocosapentaenoic acid & 19,20-EpDPE \\
\hline 13-Hydroxyoctadecadienoic acid & 13 -HODE \\
\hline 13-Hydroxyoctadecatrienoic acid & 13(S)-HOTre \\
\hline
\end{tabular}

Scientific) followed by reconstitution of the residues in a 2:1 methanol:HPLC-grade water mixture. To remove any particulates, samples were passed through Waters Acrodisc GHP $13 \mathrm{~mm}$ GHP $0.2-\mu \mathrm{m}$ syringe filters. The samples were then stored in glass chromatography vials with glass inserts and kept at $-80^{\circ} \mathrm{C}$ until quantification within 1 mo of collection. A 7-point standard curve was created with a mix of standards and the internal standards mentioned above for quantification.

All FA were quantified using a modified version of a previously described technique (Ryman et al., 2017). Briefly, reverse-phase LC on a Waters Acquity UPLC utilizing a Supelco (State College, PA) Ascentis Express $\mathrm{C} 1810 \mathrm{~cm} \times 2.1 \mathrm{~mm}, 2.7 \mu \mathrm{m}$ column was used with a flow rate of $0.35 \mathrm{~mL} / \mathrm{min}$ at $50^{\circ} \mathrm{C}$. The single quadrupole MS was in electrospray negative ionization mode, and voltage was $-3 \mathrm{kV}$ with the turbo ion spray source temperature at $450^{\circ} \mathrm{C}$. The gradient mobile phase was programmed in the following manner $(\mathrm{A} / \mathrm{B} / \mathrm{D}$ ratio): time 0 to $0.2 \mathrm{~min}(45 / 22 / 33)$, to $(80 / 19 / 1)$ at $4.0 \mathrm{~min}$ and held until $5.0 \mathrm{~min}$, to $(45 / 22 / 33)$ at $6.0 \mathrm{~min}$ and held until $8.0 \mathrm{~min}$. In this gradient mobile phase $\mathrm{A}=$ acetonitrile, $\mathrm{B}=$ methanol, and $\mathrm{D}=0.1 \%$ formic acid. Fatty acids were quantified by matching mass- 1 and retention time with corresponding deuterated internal standard abundance and calibrated to a linear 7-point standard curve $\left(\mathrm{R}^{2}>0.99\right)$ using Waters Empower 3 software (Milford, MA).

The LC-MS/MS protocol has also been reported previously by Mavangira et al. (2015). Metabolites were quantified by a Waters Acquity UPLC connected to a Waters Xevo-TQ-S tandem quadrupole mass spectrometer using multiple reaction monitoring. Chromatography separation was performed with an Ascentis Express C18 HPLC column (Sigma-Aldrich), held at $50^{\circ} \mathrm{C}$, and autosampler held at $10^{\circ} \mathrm{C}$. Mobile phase bottle A was water containing $0.1 \%$ formic acid, and mobile phase bottle $\mathrm{B}$ was acetonitrile. The flow rate was $0.3 \mathrm{~mL} /$ min. Liquid chromatography separation took $15 \mathrm{~min}$ with linear gradient steps programmed as follows (A:B ratio): time 0 to $0.5 \mathrm{~min}(99: 1)$, to $(60: 40)$ at $2.0 \mathrm{~min}$, to $(20: 80)$ at $8.0 \mathrm{~min}$, to $(1: 99)$ at $9.0 \mathrm{~min}, 0.5 \mathrm{~min}$ held at (1:99) until min 13.0; then returned to (99:1) at $13.01 \mathrm{~min}$, and held at this condition until $15.0 \mathrm{~min}$. All oxylipids were detected using electrospray ionization in negative-ion mode. Cone voltages and collision voltages were optimized for each analyte using Waters QuanOptimize software, and data analysis was carried out with Waters TargetLynx software.

\section{Statistical Analysis}

Repeated measures linear mixed effects models were constructed using the PROC MIXED procedure in SAS 
9.4. (SAS Institute Inc., Cary, NC) to assess trends in concentrations of MUFA, PUFA, SFA, and oxylipids over the study period. Each MUFA, PUFA, SFA, and oxylipid was tested in a separate model that included the fixed effect of sampling point and a random intercept for cow to account for the dependence between samples taken from the same cow. A spatial covariance residual matrix was used to account for unequal spacing between time points. Normality of residuals was visually assessed with Q-Q plots and histograms. Data were transformed either by the log or square root function that violated the normality assumption. Estimated least squares means were then back-transformed and presented as geometric means. Levene's test and graphs of predicted residuals were used to assess heteroscedasticity, which is when the variances of the residuals are unequal. Degrees of freedom were estimated using the Kenward-Rogers approximation if heteroscedasticity was present. Differences in concentrations of FA and oxylipids between different time points were tested using multiple pairwise comparisons with a Bonferroni adjustment. Statistical significance was set at $P<0.05$.

\section{RESULTS}

Table 3 shows mean concentrations of targeted MUFA, PUFA, and SFA, which are precursors to oxylipids. The MUFA analyzed in this study, oleic and palmitoleic acid, attained their respective highest concentrations at $d+2$ and +1 . All PUFA, with the exception of EPA, analyzed in this study saw numerically highest concentrations at $\mathrm{d}+2$, whereas all PUFA except ALA reached numerically lowest concentrations at $\mathrm{d}+12$. The SFA lauric acid, myristic acid, palmitic acid, and stearic acid attained the highest concentrations of the study at $\mathrm{d}+12,+6,+2$, and +2 , respectively.

Out of the 63 oxylipid species targeted, 27 were detected in this study. Of those, the majority varied over the sampling period $(P<0.05)$. Prostaglandins, a thromboxane $\left(\mathbf{T X B} \mathbf{X}_{\mathbf{2}}\right)$, and 11-HETE were representatives of the COX-derived oxylipids (Table 4). After the cessation of lactation, the prostaglandin $\mathrm{E}_{2}$ had the lowest concentrations at $\mathrm{d}+12$, which were different than all other sampling points $(P<0.01)$. Concentrations of $\mathrm{PGF}_{2 \alpha}$ were highest at $\mathrm{d}+1$. This sampling point was higher than $\mathrm{d}-6,+2$, and $+6(P<0.05)$. The COXderivatives prostaglandin $\mathrm{D}_{2}$, 6-keto-prostaglandin $\mathrm{F}_{1 \alpha}$ (a stable metabolite of prostaglandin $\mathrm{I}_{2}$ ), and $\mathrm{TXB}_{2}$ (a stable metabolite of $\mathrm{TXA}_{2}$ ) did not vary over the sampling period. The mean concentrations of 11-HETE at $\mathrm{d}+1$ were the highest of the sampling period $(P<$ $0.01)$.

The LOX-derived oxylipid concentrations for the sample period are listed in Table 5. Several LOX-derived oxylipids reached highest concentrations of the study at $1 \mathrm{~d}$ after dry-off, including 5-HETE, 5-oxoETE, 9-HODE, 13-HODE, 13-OxoODE, and 15-HETE. In contrast, the ketone derivative of 9-HODE, 9-oxoODE, reached the highest concentrations at $\mathrm{d}+2$ and did not vary over the sampling period. The highest concentrations of $13(\mathrm{~S})$-HOTrE were seen at $d+12$, which were higher compared with all other time points $(P<0.01)$. The precursor to the anti-inflammatory oxylipid protectin $\mathrm{D}_{1}, 17$-HDoHE, did not show changes throughout

Table 3. Select plasma fatty acid substrates in healthy cows $(\mathrm{n}=10)^{1}$

\begin{tabular}{|c|c|c|c|c|c|c|c|c|}
\hline Fatty acid $(\mu M)$ & \multicolumn{6}{|c|}{ Day relative to dry-off } & $\mathrm{SE}$ & $P$-value \\
\hline Arachidonic acid & $0.96^{\mathrm{b}}$ & $5.18^{\mathrm{b}}$ & $5.86^{\mathrm{ab}}$ & $26.2^{\mathrm{a}}$ & $5.15^{\mathrm{b}}$ & $0.25^{\mathrm{b}}$ & 0.38 & 0.02 \\
\hline $\mathrm{LA}^{2}$ & 290 & 1,087 & 2,860 & 5,067 & 697 & 120 & 5 & 0.04 \\
\hline $\mathrm{ALA}^{2}$ & $10.8^{\mathrm{c}}$ & $19.5^{\mathrm{bc}}$ & $39^{\mathrm{ab}}$ & $50.2^{\mathrm{a}}$ & $24.9^{\mathrm{abc}}$ & $23.2^{\mathrm{bc}}$ & 0.3 & 0.04 \\
\hline Dihomo-ALA $^{2}$ & $1.6^{\mathrm{b}}$ & $5.5^{\mathrm{b}}$ & $4^{\mathrm{ab}}$ & $25.7^{\mathrm{a}}$ & $5.64^{\mathrm{b}}$ & $0.13^{\mathrm{b}}$ & 8.38 & 0.03 \\
\hline $\mathrm{EPA}$ & $0.27^{\mathrm{b}}$ & $0.43^{\mathrm{b}}$ & $2.0^{\mathrm{a}}$ & $1.88^{\mathrm{a}}$ & $0.79^{\mathrm{b}}$ & $0.11^{\mathrm{b}}$ & 0.12 & 0.0003 \\
\hline${\text { Lauric } \text { acid }^{2}}^{2}$ & $6.88^{\mathrm{b}}$ & $9.75^{\mathrm{b}}$ & $19.1^{\mathrm{b}}$ & $14^{\mathrm{b}}$ & $15.6^{\mathrm{b}}$ & $199^{\mathrm{a}}$ & 0.06 & 0.0001 \\
\hline Myristic acid & 94 & 266 & 592 & 268 & 631 & 135 & 66.6 & 0.12 \\
\hline Oleic acid ${ }^{2}$ & $158^{\mathrm{bc}}$ & $451^{\mathrm{ab}}$ & $1,174^{\mathrm{a}}$ & $2,162^{\mathrm{a}}$ & $46^{\mathrm{c}}$ & $47^{\mathrm{c}}$ & 0.09 & 0.0002 \\
\hline Palmitic acid ${ }^{2}$ & $114^{\mathrm{c}}$ & $176^{\mathrm{c}}$ & $995^{\mathrm{ab}}$ & $1,329^{\mathrm{a}}$ & $192^{\mathrm{bc}}$ & $859^{\mathrm{a}}$ & 0.09 & 0.01 \\
\hline Palmitoleic acid & $4.26^{\mathrm{b}}$ & $14.4^{\mathrm{b}}$ & $105^{\mathrm{a}}$ & $66.7^{\mathrm{a}}$ & $10.3^{\mathrm{b}}$ & $22.1^{\mathrm{b}}$ & 4.33 & 0.0001 \\
\hline Stearic acid ${ }^{2}$ & 117 & 49.6 & 86.6 & 268 & 86.2 & 31.5 & 1.1 & 0.1 \\
\hline
\end{tabular}

${ }^{\mathrm{a}-\mathrm{d}}$ Means within a row with different superscripts are different $(P<0.05)$.

${ }^{1}$ Standard error and $P$-values listed for each row represents the SE and $P$-value for the overall ANOVA. LA $=$ linoleic acid; ALA $=\alpha$-linoleic acid; DHA = docosahexaenoic acid; DPA = docosapentaenoic acid; EPA = eicosapentaenoic acid.

${ }^{2}$ Data log or square root transformed. Back-transformed values are shown. 
Table 4. Plasma cyclooxygenase-derived oxylipids in healthy cows $(\mathrm{n}=10)^{1}$

\begin{tabular}{|c|c|c|c|c|c|c|c|c|}
\hline Oxylipid (n $M$ ) & \multicolumn{6}{|c|}{ Day relative to dry-off } & $\mathrm{SE}$ & $P$-value \\
\hline Prostaglandin $\mathrm{D}_{2}{ }^{2}$ & 0.48 & 0.33 & 0.43 & 0.2 & 0.35 & 0.3 & 0.02 & 0.46 \\
\hline $\mathrm{PGF}_{2 \alpha}$ & $0.35^{\mathrm{b}}$ & $0.7^{\mathrm{ab}}$ & $1.67^{\mathrm{a}}$ & $0.65^{\mathrm{b}}$ & $0.23^{\mathrm{b}}$ & $0.88^{\mathrm{ab}}$ & 0.22 & 0.0005 \\
\hline 6-Keto-prostaglandin $\mathrm{F}_{1 \alpha}{ }^{2}$ & 0.07 & 0.13 & 0.48 & 0.57 & 0.36 & 0.25 & 0.02 & 0.1 \\
\hline $\mathrm{TXB}_{2}{ }^{2}$ & 0.48 & 0.57 & 0.93 & 0.96 & 0.63 & 0.14 & 0.02 & 0.07 \\
\hline
\end{tabular}

${ }^{a-c}$ Means within a row with different superscripts are different $(P<0.05)$.

${ }^{1}$ Standard error and $P$-values listed for each row represent the SE and $P$-value for the overall ANOVA. TX $=$ thromboxane; HETE $=$ hydroxyeicosatetraenoic acid.

${ }^{2}$ Data log or square root transformed. Back-transformed values are shown.

the study $(P<0.13)$, achieving its numerically higher mean concentrations at $\mathrm{d}+6$.

Table 6 shows the concentrations of CYP-derived oxylipids through the sampling period. Some oxylipids produced by this pathway reached highest concentrations of the study at $\mathrm{d}+12$, whereas others reached the lowest concentrations of the study at this time. For instance, 8,9-DHET, 11,12-DHET, 14,15-DHET, 20HETE, 17,18-DiHETE, 19,20-EpDPE, and 19,20-DiHPDA attained the highest concentrations at $\mathrm{d}+12$, whereas the EpOME and DiHOME stereoisomer concentrations were lowest at this time $(P<0.05)$. Finally, the highest concentrations of 14,15-DiHETE were seen at $\mathrm{d}+2$.

\section{DISCUSSION}

The availability of FA substrates is a major determinant of oxylipid biosynthesis. Before oxylipid biosynthesis, FA are released from the $s n-2$ esterification site in the phospholipid glycerol backbone by phospholipase
$\mathrm{A}_{2}$ (Mavangira et al., 2015). Phospholipase $\mathrm{A}_{2}$ is activated in many circumstances, including after cytokine stimulation during inflammation (Schmiel and Miller, 1999). Therefore, the inflammation that occurs with mammary gland remodeling can be the inciting cause of phospholipase $\mathrm{A}_{2}$-mediated release of PUFA, leading to increased oxylipid biosynthesis at the same time. High concentrations of oleic acid throughout our study correspond to previous studies that found it to be one of the most abundant FA found in the digestive tract and blood of dairy cows (Yanting et al., 2018). The high concentrations of LA throughout the sample period are also not surprising as it has been reported as the most abundant PUFA in milk and plasma throughout lactation (Kuhn et al., 2017). Linoleic acid and other UFA are extensively hydrogenated in the rumen to produce MUFA and SFA. In fact, oleic acid was found to increase linearly with increasing concentrations of LA in vitro (Honkanen et al., 2012). Therefore, the increased concentrations of some MUFA and SFA at times of increased PUFA concentrations is anticipated.

Table 5. Plasma lipoxygenase-derived oxylipids in healthy cows $(\mathrm{n}=10)^{1}$

\begin{tabular}{|c|c|c|c|c|c|c|c|c|}
\hline Oxylipid (n $M$ ) & \multicolumn{6}{|c|}{ Day relative to dry-off } & $\mathrm{SE}$ & $P$-value \\
\hline 5 -HETE $^{2}$ & $0.52^{\mathrm{d}}$ & $1.36^{\mathrm{bcd}}$ & $3.27^{\mathrm{a}}$ & $2.48^{\mathrm{ab}}$ & $1.42^{\mathrm{bc}}$ & $1.08^{\mathrm{cd}}$ & 0.01 & 0.0001 \\
\hline 9-HODE & $7.9^{\mathrm{bc}}$ & $12.5^{\mathrm{ab}}$ & $19^{\mathrm{a}}$ & $13^{\mathrm{ab}}$ & $9.6^{\mathrm{bc}}$ & $2.19^{\mathrm{c}}$ & $1.96^{3}$ & 0.0001 \\
\hline 9-oxoODE ${ }^{2}$ & 4.35 & 5.8 & 7.19 & 9.45 & 7.66 & 8.57 & 0.06 & 0.06 \\
\hline $13-$ HODE $^{2}$ & $33.9^{\mathrm{a}}$ & $42.3^{\mathrm{a}}$ & $50.1^{\mathrm{a}}$ & $43.1^{\mathrm{a}}$ & $31.2^{\mathrm{a}}$ & $4.2^{\mathrm{b}}$ & $0.11^{3}$ & 0.0001 \\
\hline $17-\mathrm{HDoHE}^{2}$ & 1.19 & 0.9 & 1.53 & 1.42 & 1.84 & 0.76 & 0.03 & 0.13 \\
\hline
\end{tabular}

${ }^{\mathrm{a}-\mathrm{d}}$ Means within a row with different superscripts are different $(P<0.05)$.

${ }^{1}$ Standard error and $P$-values listed for each row represent the SE and $P$-value for the overall ANOVA. HETE $=$ hydroxyeicosatetraenoic acid; oxoETE = oxoeicosatetraenoic acid; HODE $=$ hydroxyoctadecadienoic acid; oxoODE $=$ oxooctadecadienoic acid; HOTrE $=$ hydroxyoctadecatrienoic acid; HDoHE = hydroxydocosahexaenoic acid.

${ }^{2}$ Data log or square root transformed. Back-transformed values were used.

${ }^{3}$ The SE for $d+1$ differed from the other sampling points and are as follows: 9-HODE (SE $\left.=2.07\right)$ and 13-HODE (SE $\left.=0.14\right)$. 
Table 6. Plasma cytochrome P450-derived oxylipids in healthy cows $(\mathrm{n}=10)^{1}$

\begin{tabular}{|c|c|c|c|c|c|c|c|c|}
\hline Oxylipid (n $M$ ) & \multicolumn{6}{|c|}{ Day relative to dry-off } & $\mathrm{SE}$ & $P$-value \\
\hline $8,9-$ DHET $^{2}$ & $0.14^{\mathrm{c}}$ & $0.2^{\mathrm{c}}$ & $0.7^{\mathrm{ab}}$ & $0.37^{\mathrm{bc}}$ & $0.24^{\mathrm{c}}$ & $1.0^{\mathrm{a}}$ & 0.01 & 0.0001 \\
\hline $14,15-\mathrm{DHET}$ & $2.21^{\mathrm{c}}$ & $4.05^{\mathrm{bc}}$ & $5.77^{\mathrm{b}}$ & $5.06^{\mathrm{b}}$ & $4.54^{\mathrm{b}}$ & $8.85^{\mathrm{a}}$ & 0.52 & 0.0001 \\
\hline $9,10-\mathrm{EpOME}^{2}$ & $2.12^{\mathrm{c}}$ & $3.0^{\mathrm{bc}}$ & $8.21^{\mathrm{a}}$ & $8.28^{\mathrm{ab}}$ & $3.34^{\mathrm{ac}}$ & $0.02^{\mathrm{d}}$ & 0.11 & 0.0001 \\
\hline $9,10-\mathrm{DiHOME}$ & $51^{\mathrm{b}}$ & $94^{\mathrm{a}}$ & $47^{\mathrm{b}}$ & $35^{\mathrm{bc}}$ & $26^{\mathrm{bc}}$ & $4.8^{\mathrm{c}}$ & 7.1 & 0.0001 \\
\hline 14,15-DiHETE & $12.2^{\mathrm{ab}}$ & $16.7^{\mathrm{ab}}$ & $22.3^{\mathrm{a}}$ & $23.7^{\mathrm{a}}$ & $19.8^{\mathrm{a}}$ & $5.88^{\mathrm{b}}$ & $3.8^{3}$ & 0.002 \\
\hline 17,18-DiHETE & $129^{\mathrm{b}}$ & $174^{\mathrm{ab}}$ & $226^{\mathrm{ab}}$ & $178^{\mathrm{ab}}$ & $194^{\mathrm{ab}}$ & $292^{\mathrm{a}}$ & $34^{3}$ & 0.01 \\
\hline $19,20-\mathrm{EpDPE}$ & $0.33^{\mathrm{b}}$ & $0.46^{\mathrm{b}}$ & $0.43^{\mathrm{b}}$ & $0.38^{\mathrm{b}}$ & $0.29^{\mathrm{b}}$ & $1.25^{\mathrm{a}}$ & 0.13 & 0.0001 \\
\hline 19,20-DiHPDA & $0.67^{\mathrm{b}}$ & $1.1^{\mathrm{b}}$ & $1.95^{\mathrm{ab}}$ & $0.94^{\mathrm{b}}$ & $1.37^{\mathrm{b}}$ & $2.42^{\mathrm{a}}$ & $0.49^{3}$ & 0.007 \\
\hline
\end{tabular}

${ }^{\mathrm{a}-\mathrm{d}}$ Means within a row with different superscripts are different $(P<0.05)$.

${ }^{1}$ Standard error and $P$-values listed for each row represent the SE and $P$-value for the overall ANOVA. DHET $=$ dihydroxyeicosatrienoic acid; EpOME = epoxyoctadecenoic acid; DiHOME = dihydroxyoctadecenoic acid; HETE $=$ hydroxyeicosatetraenoic acid; DiHETE $=$ dihydroxyeicosatetraenoic acid; DiHPDA = dihydroperoxydocosahexaenoic acid; EpDPE = epoxydocosapentaenoic acid.

${ }^{2}$ Data log or square root transformed. Back-transformed values are shown.

${ }^{3}$ The $\mathrm{SE}$ for $\mathrm{d}+1$ differed from the other sampling points and are as follows: 12,13 -EpOME $(\mathrm{SE}=0.38) ; 12,13-\mathrm{DiHOME}(\mathrm{SE}=4.7) ; 20-\mathrm{HETE}$ $(\mathrm{SE}=0.04) ; 14,15$-DiHETE $(\mathrm{SE}=3.9) ; 17,18$-DiHETE $(\mathrm{SE}=38)$; and 19,20-DiHPDA $(\mathrm{SE}=0.51)$.

Furthermore, the enzymatic pathways of oxylipid production show substrate preference, often preferring to oxidize n-3 PUFA over n-6 PUFA if they are available. The relative amounts of $n-6$ and n-3-derived oxylipids in the present study may therefore be a reflection of the concentrations of their respective PUFA precursors. Previous work has documented the alteration of oxylipid profiles in tissues by changing the dietary supply of PUFA (Raphael and Sordillo, 2013). In dairy cattle, dietary PUFA have been shown to influence the inflammatory outcomes of infectious diseases such as mastitis (Raphael and Sordillo, 2013). However, the effect of supplementing dietary n-3 PUFA on the oxylipid profile of cattle during the transition into the dry period has not been investigated and should serve as the basis for future studies.

In the present study, many of the quantified plasma oxylipids varied throughout the sample period. This is in contrast to a previous study performed on healthy cows $14 \mathrm{~d}$ prepartum to $84 \mathrm{~d}$ postpartum, which found that only 3 of the 23 measured plasma oxylipids differed throughout the sampling period (Raphael et al., 2014). However, previous work in dairy cattle has shown that oxylipid biosynthesis is complex, varying within a lactation. For instance, PUFA and total oxylipids in bovine milk were lower in early lactation than during mid or late lactation (Kuhn et al., 2017). In fact, it would be reasonable to think that the oxylipid profile of high-producing cows may differ from that of low-producing cows. However, our group found no differences in concentrations of other serum inflammatory biomarkers, such as acute phase proteins, between high- and low-producing cows during the early dry period (Putman et al., 2018). Therefore, we suggest that milk production may not affect oxylipid concentrations in the early dry period as they are also related to inflammation. Additionally, pro- and anti-inflammatory oxylipids were not altered to the same degree. Whereas the pro-inflammatory 20-HETE was increased in the periparturient period, other pro-inflammatory oxylipids like 9,10-DiHOME and 5-oxoETE were decreased compared with mid and late lactation (Kuhn et al., 2017). That being said, many of the oxylipids in the present study reached their highest concentrations at $d$ $+1,+2$, or +12 , regardless of being typically considered pro- or anti-inflammatory (Figure 2). This pattern may be explained by the inflammation that occurs during early involution to adjust the mammary gland from a lactating to a nonlactating state because oxylipid production follows the onset of inflammation (Sordillo, 2018). This is supported by the fact that other biomarkers of inflammation in serum, such as nonesterified FA, albumin, and haptoglobin, suggested that dairy cattle undergo inflammation in the early dry period at $\mathrm{d}+1$ and +12 in a previous study completed by our group (Putman et al., 2018). Additional support that the inflammation required by mammary involution may be related to oxylipid concentrations is provided because neutrophils and macrophages are abundant in the mammary gland during acute involution (Sordillo and Nickerson, 1988). Indeed, the inflammatory cells present during acute involution are likely contributing to the oxylipid profile noted in the present study as they are known to produce oxylipids, including prosta- 


\begin{tabular}{|c|c|c|c|c|c|}
\hline$d-6$ & $\mathrm{~d} 0$ & $\mathrm{~d} 1$ & $\mathrm{~d} 2$ & d 6 & d 12 \\
\hline - $\mathrm{PGD}_{2}$ & - DiHOME & $\begin{array}{ll}\text { - } & \mathrm{PGF}_{2 \alpha} \\
\text { - } & \text { HETE (11-, 5-, } \\
& 15-) \\
\text { - } & \text { 5-OXOETE } \\
\text { - } & \text { HODE } \\
\text { - } & \text { 13-OXOODE }\end{array}$ & $\begin{array}{ll}\text { - } & \mathrm{PGE}_{2} \\
\text { - } & \text { 6-keto-PGF } \\
\text { - } & \mathrm{TXB}_{2} \\
\text { - } & \text { 9-oxoODE } \\
\text { - } & \text { EpOME } \\
\text { - } & 14,15-\mathrm{DiHETE}\end{array}$ & - 17-HDoHE & 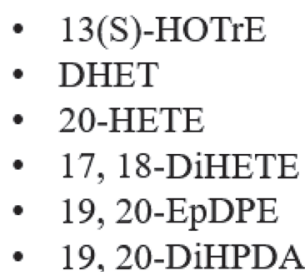 \\
\hline
\end{tabular}

Figure 2. Sampling point at which each oxylipid attained their respective highest concentration. DiHOME $=$ dihydroxyoctadecenoic acid; HETE $=$ hydroxyeicosatetraenoic acid; oxoETE $=$ oxoeicosatetraenoic acid; HODE $=$ hydroxyoctadecadienoic acid; oxoODE $=$ oxooctadecadienoic acid; TX = thromboxane; DiHETE $=$ dihydroxyeicosatetraenoic acid; DHET $=$ dihydroxyeicosatrienoic acid; HOTrE $=$ hydroxyoctadecatrienoic acid; EpOME = epoxyoctadecaenoic acid; HDoHE = hydroxydocosahexaenoic acid; EpDPE = epoxydocosapentaenoic acid; DiHPDA $=$ dihydroperoxydocosahexaenoic acid.

glandins (Dennis and Norris, 2015), 15-HETE (Sordillo, 2018), and EpOME (Thompson and Hammock, 2007). The influence cell type has on oxylipid biosynthesis is partially due to the enzymes they possess. For example, platelets are capable of producing thromboxanes from arachidonic acid because they contain COX enzymes whereas reticulocytes can produce certain HETE due to the presence of LOX (Kuhn et al., 2002; Courtois et al., 2018). Under the influence of different pathways, a given substrate can be metabolized into a variety of oxylipids that can perform diverse functions.

Oxylipids produced via the COX pathway include numerous prostaglandins and thromboxanes that are capable of a wide range of biological activities. For instance, prostaglandin $\mathrm{D}_{2}$ is associated with significant anti-inflammatory actions, whereas prostaglandin $\mathrm{E}_{2}$ is associated with pro-inflammatory actions in the gastrointestinal tract (Wallace, 2018). In a mouse model of lung cancer, some prostaglandins were increased at earlier time points than others. Poczobutt et al. (2013) found that $\mathrm{PGF}_{2 \alpha}$ and prostaglandin $\mathrm{E}_{2}$ were increased at 2 wk after cancer cell injection, whereas prostaglandin $\mathrm{D}_{2}$ and $\mathrm{TXB}_{2}$ were increased 3 wk after injection. The authors suggested this time dependency could be due to changing phenotypes of inflammatory cells, such as macrophages, during tumor progression (Poczobutt et al., 2013). Similarly, COX-derived oxylipids in the present study were increased at different times. In mice, the phenotype of macrophages and mammary epithelial cells transforms to the M2 phenotype during mammary involution (Hughes et al., 2012). Therefore, the phenotype of the cells present in the early dry period of dairy cows may be affecting the timing of oxylipid biosynthesis in this study as well. Although 11-HETE can be produced via COX enzymes, it is typically associated with nonenzymatic production through interactions between lipid membranes and ROS. The effects of 11-HETE have been reported as potently pro-inflammatory, vaso- constrictive, and immunosuppressive (Ferreiro-Vera et al., 2011). In the present study, 11-HETE was increased at $d+1$, which is a sampling point where our previous study found that ROS production was also increased (Putman et al., 2018), and therefore may be a result of increased nonenzymatic production at this time.

Likewise, LOX-derived oxylipids display a wide range of biological actions. For instance, 15-HETE has been associated with anti-inflammatory effects, whereas 9and 13-HODE have been associated with anti-thrombotic effects in humans (Wolfer et al., 2017). In accordance with a study performed by Raphael et al. (2014), plasma concentrations of 9- and 13-HODE followed a similar trend in healthy cows across the sample period. However, the concentrations of 13-HODE were at least 2 -fold higher than 9-HODE at all sampling points in the present study, which is in contrast to the Raphael et al. (2014) study where 9-HODE concentrations were at least 2-fold higher than 13-HODE. Many functions have been attributed to HODE, both pro- and anti-inflammatory. For example, HODE has been implicated in pro-inflammatory actions via the induction of adhesion molecule expression on human vascular tissue but also in the activation of peroxisome proliferator-activated receptors, which serves as an anti-inflammatory action (Ricote et al., 1998; Friedrichs et al., 1999). However, the role of 9- and 13-HODE during the early dry period in dairy cattle is still unknown and needs to be investigated further. Both 9- and 13-HODE can be oxidized to form the ketone derivatives 9- and 13-oxoODE, which also have shown both pro-inflammatory effects by activating pain receptors and anti-inflammatory effects by acting as a ligand for peroxisome proliferator-activated receptors (Mattmiller et al., 2014). Two LOX-derived oxylipids associated with anti-inflammatory outcomes, 13(S)-HOTrE and 17-HDoHE (a precursor to an antiinflammatory oxylipid, protectin D1), were increased at differing time points in the present study. Both 
13(S)-HOTrE and 17-HDoHE were found to increase in a mouse model during the recovery phase of inflammatory bowel disease (Hamabata et al., 2018). Although the presence of these compounds in our study suggests anti-inflammatory properties, the differential times at which they were increased further supports that oxylipid biosynthesis is complex and more research needs to be completed to determine the cause of differential oxylipid production during early involution. For instance, it is still difficult to distinguish if and when oxylipids are the cause of or merely a consequence of inflammation and this would be beneficial to investigate in the future. Such studies may involve noting if an inflammatory response occurs upon stimulation with certain oxylipids.

Following suit with the other enzymatic pathways, CYP-derived oxylipids can also exert a wide range of biological effects. For instance, the epoxy metabolites of n-3 PUFA have demonstrated potent anti-inflammatory effects and play an important role in vascular homeostasis (Heemskerk et al., 2014). The DHA-derived oxylipid, 19,20-EpDPE, exemplifies this point as it has been found to have potent vasodilatory effects. Furthermore, the epoxy metabolites can be further metabolized into corresponding vicinal diols, such as 19,20-DiHPDA, via soluble epoxide hydrolase (Fischer et al., 2014). The diols produced often have differing potencies and functions than their epoxy substrate. For instance, DHET and 19,20-DiHPDA are less biologically active than epoxyeicosatrienoic acid and 19,20-EpDPE, respectively (Heemskerk et al., 2014; Duflot et al., 2017). In the present study, the LA-derived epoxide products 9,10- and 12,13 -EpOME reached the highest concentrations at $\mathrm{d}$ +2 whereas the epoxide hydration products $(9,10-$ and 12,13-DiHOME) had the highest concentrations at $\mathrm{d} 0$. This may suggest that the epoxides are being rapidly converted to their respective diols at $\mathrm{d} 0$, whereas the conversion may be dampened at $\mathrm{d}+2$. Utilizing soluble epoxide hydrolase inhibitors to prolong the availability of the anti-inflammatory epoxides has been proposed as a therapy for pathologies such as inflammatoryrelated pain, atherosclerosis, and metabolic syndrome in humans (Swardfager et al., 2018). However, these inhibitors have not been investigated in dairy cattle, so whether or not they would be a beneficial therapy for modulating inflammatory responses is unknown. Pro-inflammatory actions are performed by 20-HETE, which is able to increase ROS production (Han et al., 2013). In bovine cells, 20-HETE has been associated with vasodilation and increased production of superoxide anion and hydrogen peroxide (Bodiga et al., 2010). Additionally, hydrogen peroxide has been shown to activate the $\mathrm{Ca}^{2+}$ /calmodulin-dependent protein kinase II/Janus-kinase 2-dependent pathway in bovine aortic endothelial cells (Bodiga et al., 2010). Janus-kinase 2 is critical for the proliferation and survival of mammary epithelial cells (Creamer et al., 2010) and perhaps the increased concentrations of 20-HETE in this study are associated with mammary gland remodeling through a similar mechanism. The arachidonic acid-derived 14,15and 17,18-DiHETE are known for anti-inflammatory actions. For instance, 14,15-DiHETE has been shown to reduce superoxide anion generation (Vachier et al., 2002). The increased concentrations of these oxylipids at $\mathrm{d}+1$ in the study, therefore, may be the result of anti-inflammatory compounds being produced in response to inflammation.

\section{CONCLUSIONS}

Overall, oxylipids varied throughout the transition into early mammary gland involution. However, the oxylipid profile of this time diverged from those observed previously at different stages of the lactation cycle or in cows with differing health status. This study documented the oxylipids present during the early dry period for the first time, which contributes valuable knowledge given the diverse actions the potent inflammatory mediators can perform. However, it remains unknown whether the presence or alterations of these oxylipids during this time affect cow health and productivity. Therefore, future studies should be directed toward investigating causes of the changes noted along with determining if these oxylipids have an effect on cow health and productivity throughout the lactation cycle.

\section{ACKNOWLEDGMENTS}

This study was funded, in part, by support from the Agriculture and Food Research Initiative Competitive Grants Program (2014-68004-21972 and 2017-6701526676) from the USDA National Institute of Food and Agriculture (NIFA), an endowment from the Matilda R. Wilson Fund (Detroit, MI), and the Michigan Alliance for Animal Agriculture. This material also is based on work supported by the USDA NIFA under award number 2017-38420-26759. We thank the Michigan State University Mass Spectrometry and Metabolomics Core for their assistance in liquid chromatography.

\section{REFERENCES}

Akers, R. M., and S. C. Nickerson. 2011. Mastitis and its impact on structure and function in the ruminant mammary gland. J. Mammary Gland Biol. Neoplasia 16:275-289. https://doi.org/10.1007/ s10911-011-9231-3.

Altmann, R., M. Hausmann, T. Spöttl, M. Gruber, A. W. Bull, K. Menzel, D. Vogl, H. Herfarth, J. Schölmerich, W. Falk, and G. 
Rogler. 2007. 13-Oxo-ODE is an endogenous ligand for PPAR $\gamma$ in human colonic epithelial cells. Biochem. Pharmacol. 74:612-622.

Atabai, K., D. Sheppard, and Z. Werb. 2007. Roles of the innate immune system in mammary gland remodeling during involution. J. Mammary Gland Biol. Neoplasia 12:37-45. https://doi.org/10 $.1007 / \mathrm{s} 10911-007-9036-6$.

Bodiga, S., S. K. Gruenloh, Y. Gao, V. L. Manthati, N. Dubasi, J. R. Falck, M. Medhora, and E. R. Jacobs. 2010. 20-HETE-induced nitric oxide production in pulmonary artery endothelial cells is mediated by NADPH oxidase, $\mathrm{H}_{2} \mathrm{O}_{2}$, and PI3-kinase/Akt. Am. J. Physiol. Lung Cell. Mol. Physiol. 298:L564-L574. https://doi.org/ 10.1152/ajplung.00298.2009.

Bradley, A. J., and M. J. Green. 2004. The importance of the nonlactating period in the epidemiology of intramammary infection and strategies for prevention. Vet. Clin. North Am. Food Anim. Pract. 20:547-568. https://doi.org/10.1016/j.cvfa.2004.06.010.

Buczynski, M. W., D. S. Dumlao, and E. A. Dennis. 2009. Thematic Review Series: Proteomics. An integrated omics analysis of eicosanoid biology. J. Lipid Res. 50:1015-1038. https://doi.org/10 .1194/jlr.R900004-JLR200.

Calder, P. C. 2009. Polyunsaturated fatty acids and inflammation: New twists in an old tale. Biochimie 91:791-795. https://doi.org/ 10.1016/j.biochi.2009.01.008.

Courtois, A., G. Makrygiannis, J. P. Cheramy-Bien, A. Purnelle, B. Pirotte, J. M. Dogne, J. Hanson, J. O. Defraigne, P. Drion, and N. Sakalihasan. 2018. Therapeutic applications of prostaglandins and thromboxane A2 inhibitors in abdominal aortic aneurysms. Curr. Drug Targets. https://doi.org/10.2174/ 1389450119666171227224314.

Creamer, B. A., K. Sakamoto, J. W. Schmidt, A. A. Triplett, R. Moriggl, and K. U. Wagner. 2010. Stat5 promotes survival of mammary epithelial cells through transcriptional activation of a distinct promoter in Akt1. Mol. Cell. Biol. 30:2957-2970. https:// doi.org/10.1128/MCB.00851-09.

Dennis, E. A., and P. C. Norris. 2015. Eicosanoid storm in infection and inflammation. Nat. Rev. Immunol. 15:511-523. https://doi .org/10.1038/nri3859.

Duflot, T., T. Pereira, C. Roche, M. Iacob, P. Cardinael, N. E. Hamza, C. Thuillez, P. Compagnon, R. Joannides, F. Lamoureux, and J. Bellien. 2017. A sensitive LC-MS/MS method for the quantification of regioisomers of epoxyeicosatrienoic and dihydroxyeicosatrienoic acids in human plasma during endothelial stimulation. Anal. Bioanal. Chem. 409:1845-1855. https://doi.org/10.1007/s00216 -016-0129-1.

Ferreiro-Vera, C., J. M. Mata-Granados, F. Priego-Capote, J. M. Quesada-Gomez, and M. D. Luque de Castro. 2011. Automated targeting analysis of eicosanoid inflammation biomarkers in human serum and in the exometabolome of stem cells by SPE-LCMS/MS. Anal. Bioanal. Chem. 399:1093-1103. https://doi.org/10 .1007/s00216-010-4400-6.

Fischer, R., A. Konkel, H. Mehling, K. Blossey, A. Gapelyuk, N. Wessel, C. von Schacky, R. Dechend, D. N. Muller, M. Rothe, F. C. Luft, K. Weylandt, and W. H. Schunck. 2014. Dietary omega-3 fatty acids modulate the eicosanoid profile in man primarily via the CYP-epoxygenase pathway. J. Lipid Res. 55:1150-1164. https: //doi.org/10.1194/jlr.M047357.

Friedrichs, B., M. Toborek, B. Hennig, L. Heinevetter, C. Müller, and R. Brigelius-Flohé. 1999. 13-HPODE and 13-HODE modulate cytokine induced expression of endothelial cell adhesion molecules differently. Biofactors 9:61-72.

Gabbs, M., S. Leng, J. G. Devassy, M. Monirujjaman, and H. M. Aukema. 2015. Advances in our understanding of oxylipins derived from dietary PUFAs. Adv. Nutr. 6:513-540. https://doi.org/ 10.3945/an.114.007732

Hamabata, T., T. Nakamura, S. Masuko, S. Maeda, and T. Murata. 2018. Production of lipid mediators across different disease stages of dextran sulfate sodium-induced colitis in mice. J. Lipid Res. https://doi.org/10.1194/jlr.M079095.

Han, Y., H. Zhao, H. Tang, X. Li, J. Tan, Q. Zeng, and C. Sun. 2013. 20-Hydroxyeicosatetraenoic acid mediates isolated heart ischemia/ reperfusion injury by increasing NADPH oxidase-derived reactive oxygen species production. Circ. J. 77:1807-1816. https://doi.org/ 10.1253/circj.CJ-12-1211.

Heemskerk, M. M., H. K. Dharuri, S. A. van den Berg, H. S. Jonasdottir, D. P. Kloos, M. Giera, K. W. van Dijk, and V. van Harmelen. 2014. Prolonged niacin treatment leads to increased adipose tissue PUFA synthesis and anti-inflammatory lipid and oxylipin plasma profile. J. Lipid Res. 55:2532-2540. https://doi.org/10.1194/jlr .M051938.

Honkanen, A. M., J. M. Griinari, A. Vanhatalo, S. Ahvenjarvi, V Toivonen, and K. J. Shingfield. 2012. Characterization of the disappearance and formation of biohydrogenation intermediates during incubations of linoleic acid with rumen fluid in vitro. J. Dairy Sci. 95:1376-1394.

Hughes, K., J. A. Wickenden, J. E. Allen, and C. J. Watson. 2012. Conditional deletion of Stat3 in mammary epithelium impairs the acute phase response and modulates immune cell numbers during post-lactational regression. J. Pathol. 227:106-117. https://doi .org/10.1002/path.3961.

Kuhn, H., M. Walther, and R. J. Kuban. 2002. Mammalian arachidonate 15-lipoxygenases structure, function, and biological implications. Prostaglandins Other Lipid Mediat. 68-69:263-290. https:/ /doi.org/10.1016/S0090-6980(02)00035-7.

Kuhn, M. J., V. Mavangira, J. C. Gandy, C. Zhang, A. D. Jones, and L. M. Sordillo. 2017. Differences in the oxylipid profiles of bovine milk and plasma at different stages of lactation. J. Agric. Food Chem. 65:4980-4988. https://doi.org/10.1021/acs.jafc.7b01602.

Mattmiller, S. A., B. A. Carlson, J. C. Gandy, and L. M. Sordillo. 2014. Reduced macrophage selenoprotein expression alters oxidized lipid metabolite biosynthesis from arachidonic and linoleic acid. J. Nutr. Biochem. 25:647-654. https://doi.org/10.1016/j .jnutbio.2014.02.005.

Mavangira, V., J. C. Gandy, C. Zhang, V. E. Ryman, A. Daniel Jones, and L. M. Sordillo. 2015. Polyunsaturated fatty acids influence differential biosynthesis of oxylipids and other lipid mediators during bovine coliform mastitis. J. Dairy Sci. 98:6202-6215. https://doi .org/10.3168/jds.2015-9570.

Poczobutt, J. M., M. Gijon, J. Amin, D. Hanson, H. Li, D. Walker, M. Weiser-Evans, X. Lu, R. C. Murphy, and R. A. Nemenoff. 2013. Eicosanoid profiling in an orthotopic model of lung cancer progression by mass spectrometry demonstrates selective production of leukotrienes by inflammatory cells of the microenvironment. PLoS One 8:e79633. https://doi.org/10.1371/journal.pone.0079633.

Putman, A. K., J. L. Brown, J. C. Gandy, L. Wisnieski, and L. M. Sordillo. 2018. Changes in biomarkers of nutrient metabolism, inflammation, and oxidative stress in dairy cows during the transition into the early dry period. J. Dairy Sci. 101:9350-9359. https: //doi.org/10.3168/jds.2018-14591.

Raphael, W., L. Halbert, G. A. Contreras, and L. M. Sordillo. 2014. Association between polyunsaturated fatty acid-derived oxylipid biosynthesis and leukocyte inflammatory marker expression in periparturient dairy cows. J. Dairy Sci. 97:3615-3625.

Raphael, W., and L. M. Sordillo. 2013. Dietary polyunsaturated fatty acids and inflammation: The role of phospholipid biosynthesis. Int. J. Mol. Sci. 14:21167-21188. https://doi.org/10.3390/ ijms141021167.

Rastani, R. R., R. R. Grummer, S. J. Bertics, A. Gümen, M. C. Wiltbank, D. G. Mashek, and M. C. Schwab. 2005. Reducing dry period length to simplify feeding transition cows: Milk production, energy balance, and metabolic profiles. J. Dairy Sci. 88:1004-1014.

Ricote, M., A. C. Li, T. M. Wilson, C. J. Kelly, and C. K. Glass. 1998. The peroxisome proliferator-activated receptor- $\gamma$ is a negative regulator of macrophage activation. Nature 391:79-82.

Ryman, V. E., N. Packiriswamy, B. Norby, S. E. Schmidt, A. L. Lock, and L. M. Sordillo. 2017. Supplementation of linoleic acid (C18:2n -6) or alpha-linolenic acid (C18:3n-3) changes microbial agonistinduced oxylipid biosynthesis. J. Dairy Sci. 100:1870-1887. https:/ /doi.org/10.3168/jds.2016-11599.

Schmiel, D. H., and V. L. Miller. 1999. Bacterial phospholipases and pathogenesis. Microbes Infect. 1:1103-1112. https://doi.org/10 .1016/S1286-4579(99)00205-1. 
Smith, G. L., N. C. Friggens, C. J. Ashworth, and M. G. G. Chagunda. 2017. Association between body energy content in the dry period and post-calving production disease status in dairy cattle. Animal 11:1590-1598. https://doi.org/10.1017/S1751731117000040.

Sordillo, L. M. 2018. Symposium review: Oxylipids and the regulation of bovine mammary inflammatory responses. J. Dairy Sci. 101:5629-5641.

Sordillo, L. M., and S. C. Nickerson. 1988. Morphologic changes in the bovine mammary gland during involution and lactogenesis. Am. J. Vet. Res. 49:1112-1120.

Swardfager, W., M. Hennebelle, D. Yu, B. D. Hammock, A. J. Levitt, K. Hashimoto, and A. Y. Taha. 2018. Metabolic/inflammatory/ vascular comorbidity in psychiatric disorders; soluble epoxide hydrolase $(\mathrm{sEH})$ as a possible new target. Neurosci. Biobehav. Rev. 87:56-66. https://doi.org/10.1016/j.neubiorev.2018.01.010.

Thompson, D. A., and B. D. Hammock. 2007. Dihydroxyoctadecamonoenoate esters inhibit the neutrophil respiratory burst. J. Biosci. 32:279-291.
Vachier, I., P. Chanez, C. Bonnans, P. Godard, J. Bousquet, and C. Chavis. 2002. Endogenous anti-inflammatory mediators from arachidonate in human neutrophils. Biochem. Biophys. Res. Commun. 290:219-224. https://doi.org/10.1006/bbrc.2001.6155.

Wallace, J. L. 2018. Eicosanoids in the GI tract. Br. J. Pharmacol. https://doi.org/10.1111/bph.14178. In press.

Wolfer, A. M., A. J. Scott, C. Rueb, M. Gaudin, A. Darzi, J. K. Nicholson, E. Holmes, and J. M. Kinross. 2017. Longitudinal analysis of serum oxylipin profile as a novel descriptor of the inflammatory response to surgery. J. Transl. Med. 15:83. https://doi.org/10 .1186/s12967-017-1171-2.

Yanting, C., Q. Y. Yang, G. L. Ma, M. Du, J. H. Harrison, and E. Block. 2018. Dose- and type-dependent effects of long-chain fatty acids on adipogenesis and lipogenesis of bovine adipocytes. J. Dairy Sci. 101:1601-1615. https://doi.org/10.3168/jds.2017-13312.

Zobel, G., D. M. Weary, K. E. Leslie, and M. A. von Keyserlingk. 2015. Invited review: Cessation of lactation: Effects on animal welfare. J. Dairy Sci. 98:8263-8277. https://doi.org/10.3168/jds.2015-9617. 